\title{
Exchange Dynamics of Dynamin Measured in Living Cells During Endocytic Vesicle Formation
}

\author{
Léa Claverie ${ }^{1,2}$, Morgane Rosendale ${ }^{1,2}$, Patrice Maschalchi ${ }^{3}$, Corey Butler ${ }^{1,2}$, Natacha Retailleau ${ }^{1,2}$, \\ Jean-Baptiste Sibarita ${ }^{1,2}$, Daniel Choquet ${ }^{1,2,3}$ \& David Perrais ${ }^{1,2^{*}}$ \\ 1. University of Bordeaux, Interdisciplinary Institute for Neuroscience, UMR 5297, F-33000 \\ Bordeaux, France \\ 2. CNRS, Interdisciplinary Institute for Neuroscience, UMR 5297, F-33000 Bordeaux, France. \\ 3. Bordeaux Imaging Center, UMS 3420 CNRS, US4 INSERM, University of Bordeaux, Bordeaux, \\ France. \\ *Corresponding author: david.perrais@u-bordeaux.fr
}

During clathrin mediated endocytosis (CME) at the plasma membrane, the GTPase dynamin is recruited to the neck of nascent clathrin-coated vesicles (CCVs) where it oligomerizes into helical filaments. Conformational changes induced by the hydrolysis of GTP catalyze the scission of the vesicle neck. This process has been studied in great detail with in vitro reconstitution on membrane tubules $[1,2]$ but it needs to be established in living cells, where dynamin interactions with SH3 domain containing proteins such as amphiphysin are critical [3]. Live cell TIRF imaging with the ppH assay [4] allows the detection of CCV formation with high spatial $(\sim 100 \mathrm{~nm})$ and temporal $(2 \mathrm{~s})$ resolutions. It has revealed that dynamin is recruited to maturing clathrin coated pits (CCPs) in two phases with a peak at the time of scission [5] but the parameters of its recruitment in living cells remain unclear.

To determine these parameters, we have performed live cell imaging of dynamin recruitment at collective and single molecule levels during acute perturbations of its function. First, we showed that Dyngo4a, a cell permeable blocker of dynamin GTPase activity, or GTP $\gamma \mathrm{S}$ dialyzed through a patchclamp pipette, quickly blocked CME and led to the accumulation of dynamin-mCherry at CCPs. Partial block decreased the rate of dynamin recruitment before CCV formation, suggesting that GTPase activity regulates its recruitment at early stages of $\mathrm{CCV}$ formation. To avoid any effect of diffusion, we have performed photo-activation of DMNPE-GTP $\gamma \mathrm{S}$ : this compound is inactive before UV illumination, but blocks endocytosis within $4 \mathrm{~s}$ while provoking dynamin accumulation on a longer timescale. We next investigated the parameters of dynamin recruitment to forming CCVs. FRAP analysis showed that dynamin exchange is fast and complete and was only moderately impaired by saturating concentrations of Dyngo4a, suggesting that dynamin exchanges with an extra-CCP pool at all times, including association in the oligomeric helix around the tubular neck.

To get better insight into the modes of dynamin recruitment at all stages of CCP maturation we conducted dual $\mathrm{ppH} /$ single protein tracking photo-activation localization microscopy (sptPALM) imaging in cells expressing dynamin-mEos3.2 (Figure 1). Dynamin is recruited to the plasma membrane, diffuses outside of CCPs and is trapped at CCPs, as revealed by the existence of a large number of trajectories outside of CCPs (Figure 1A) but its diffusion is slower within CCPs (Figure 1B). The number of detected molecules increases as scission approaches (Figure 1C) but single molecules are equally immobilized at all stages of CCP maturation. We conclude that dynamin exchanges with an extra-CCP pool at all times (Figure 1D): this would allow for its further recruitment by addition of new binding sites and its ability to narrow the vesicle neck after GTP hydrolysis [6]. 


\section{References:}

[1] Dar S, Kamerkar SC, Pucadyil TJ. A high-throughput platform for real-time analysis of membrane fission reactions reveals dynamin function. Nat Cell Biol 17:1588-1596 (2015)

[2] Kong L, Sochacki KA, Wang H, Fang S, Canagarajah B, Kehr AD, et al. Cryo-EM of the dynamin polymer assembled on lipid membrane. Nature 560:258-262 (2018)

[3] Shupliakov O, Löw P, Grabs D, Gad H, Chen H, David C, et al. Synaptic vesicle endocytosis impaired by disruption of dynamin-SH3 domain interactions. Science 276:259-263 (1997)

[4] Merrifield CJ, Perrais D, Zenisek D. Coupling between Clathrin-Coated-Pit Invagination, Cortactin Recruitment, and Membrane Scission Observed in Live Cells. Cell 121:593-606 (2005)

[5] Taylor MJ, Perrais D, Merrifield CJ. A High Precision Survey of the Molecular Dynamics of Mammalian Clathrin-Mediated Endocytosis. Schmid SL, editor. PLoS Biol 9:e1000604 (2011)

[6] The authors acknowledge funding from the Centre National de la Recherche Scientifique, the Agence Nationale de la Recherche (ANR-12-BSV5-0005 "CaPeBIE") and the Aquitaine Regional Council.
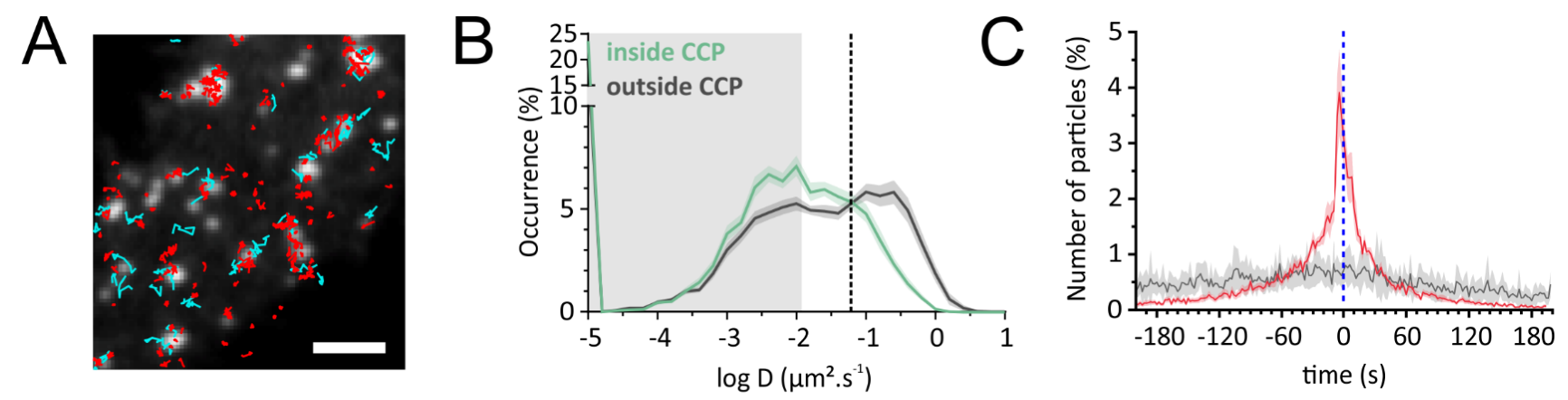

D Dynamin

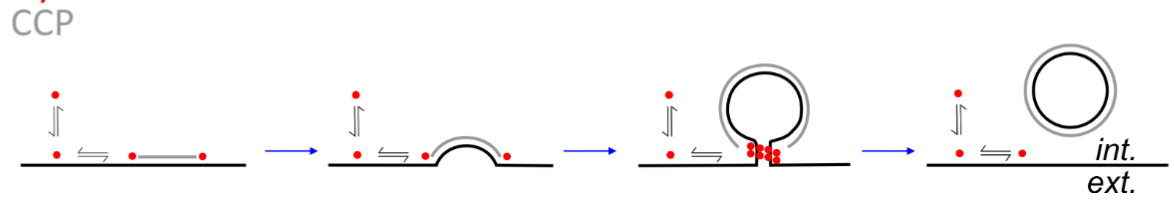

Figure 1: Imaging of single dynamin proteins at the surface of living cells. A. Portion of an NIH 3 T3 cell transfected with TfR-SEP and dynamin1-mEOS3.2 and imaged with TIRF microscopy with time lapse at $0.25 \mathrm{~Hz}$ (for TfR-SEP) and sptPALM at $50 \mathrm{~Hz}$ (for dyn1-mEOS3.2). The image of CCPs labelled with TfR-SEP is overlaid with trajectories of single dynamin1-mEOS3.2 molecules. Red lines show trajectories of single molecules classified as immobile $\left(\mathrm{D}<10^{-2} \mu \mathrm{m}^{2} \cdot \mathrm{s}^{-1}\right)$ and cyan lines, single molecules classified as diffusive $\left(\mathrm{D}>10^{-2} \mu \mathrm{m}^{2} \cdot \mathrm{s}^{-1}\right)$. Scale bar $2 \mu \mathrm{m}$. B. Distribution of diffusion coefficients measured inside CCPs (green curve) and outside CCPs (black curve) for 31 cells (4 independent experiments). C. Normalized number of particles in CCPs relative to the time of scission measured with the $\mathrm{ppH}$ protocol. Red, real data (10 cells); grey, randomized data. D. Model of dynamin recruitment to CCPs at all stages of vesicle formation. 